\title{
Diagnostic Accuracy of Sonosalpingography for Assessing Tubal Pathology in Women with Secondary Infertility Taking Laparoscopy as Gold Standard
}

\author{
Amit Nandan Dhar Dwivedi*, Madhu Jain, Suchi Tripathi, Sunny Garg, Shivi Jain \\ Institute of Medical Sciences, Banaras Hindu University, Varanasi, India \\ Email: ${ }^{*}$ amitnandan21@yahoo.com
}

Received June 22, 2012; revised July 21, 2012; accepted July 31, 2012

\begin{abstract}
Context: Secondary infertility is a major public health issue with growing urbanisation and increasing age of marriage among women. Disease limited to low socioeconomic strata is commonly seen in general population. Judicious and accurate assessment of underlying pathology targeting tubes should be undertaken. Aims: The aim of this observational study was to assess the diagnostic accuracy of sonosalpingography taking laparoscopy as gold standard in patients of secondary infertility. Main aim was to assess tubal factors as an underlying cause and to assess feasibility of a radiation free protocol in patients of reproductive age group. Settings and Design: The study was carried out in a hospital setting. A total of 123 patients were selected. Patients of primary infertility were excluded. Sonosalpingography was performed by a single experienced radiologist. This was confirmed by laparoscopy conducted by an experienced gynaecologist. Methods and Material: A detailed clinical and physical examination including internal examination was done. This was followed by sonosalpingography using high frequency TVS probe (Philips IU 22). All patients were subjected to laparoscopic evaluation on the same day. Statistical Analysis: Sensitivity, specificity, diagnostic accuracy and predictive values. Results: Sonosalpingography was $72.91 \%$ sensitive, $81.33 \%$ specific with diagnostic accuracy of $78.05 \%$ and positive predictive value of $71.42 \%$ for tubal pathologies. Conclusions: Sonosalpingography is a very useful complementary diagnostic modality in assessment of tubal pathology. This should precede direct laparoscopic evaluation. Patients of secondary infertility can be assessed with a combination of sonosalpingography and laparoscopy. This saves the patient of radiation exposure as well as pain of contrast administration.
\end{abstract}

Keywords: Sonosalpingography; Secondary Infertility; Laparoscopy; Chromopertubation

\section{Introduction}

Patients of infertility are burdened with unnecessary work up and diagnostic protocol. This study was conducted in wake of rising cases of secondary infertility due to tubal factors. Conventional hysterosalpingogram (HSG) has been the main stay for outlining tubal lumen and uterine cavity. With the advent of transvaginal sonography (TVS) endometrial pathologies can be accurately diagnosed. This study was designed to see if a combination of sonosalpingography along with gold standard laparoscopy could supplant conventional HSG. The need of contrast administration and radiation exposure was obviated keeping young females as a target group. According to recent estimates, the average person in the US receives an effective dose of about $3 \mathrm{mSv}$ per year from naturally occurring radioactive materials and cosmic radiation from outer space. These natural "background" doses vary throughout

"Corresponding author. the country. Average entrance surface dose for conventional HSG is $13.3 \mathrm{mSv}$ with additional life time risk of developing a fatal cancer being low. The aim of this observational study was to assess the diagnostic accuracy of sonosalpingography taking laparoscopy as gold standard in patients of secondary infertility and to assess tubal factors as an underlying cause.

\section{Subjects and Methods}

In this study one hundred twenty three patients (123) patients complaining of secondary infertility were selected. Patients who had uterine pathologies like fibroids active pelvic inflammatory disease, endometrial polyps, Asherman's syndrome etc. were excluded. Patients of primary infertility were excluded. Detailed menstrual and obstetrical history was obtained. This was followed by physical and gynaecological examination. Taking lead from history and examination, suitable patients were advised sonosalpingography on day nine of menstrual period. Informed 
verbal consent was taken. Thirty minutes prior to the examination the patient was given mild analgesic (Dicyclomine/im). The patient was subjected to pre-procedural TVS scanning. The cervix was exposed using a speculum and anterior lip of cervix held by a tenaculum. A 10 Fr Foley's catheter was inserted in the uterine cavity and balloon was inflated to secure position (Figure 1). The probe was inserted to check position. The tenaculum was removed and $20 \mathrm{ml}$ of normal saline was administered for each side. The passage of saline through the tube was observed real time (Figure 2). We did not use colour Doppler, 3D reformation or any ultrasound contrast agents. After the procedure catheter was removed and post procedural scan was done. The patient was comfortably discharged.

The gynaecologist was blinded about the results of sonosalpingography. All patients irrespective of findings of sonosalpingography underwent laparoscopic evaluation on the same day. Both adnexa were examined. Ovaries were assessed for its morphology and other findings. The tube was assessed till the fimbrial aspect. This was

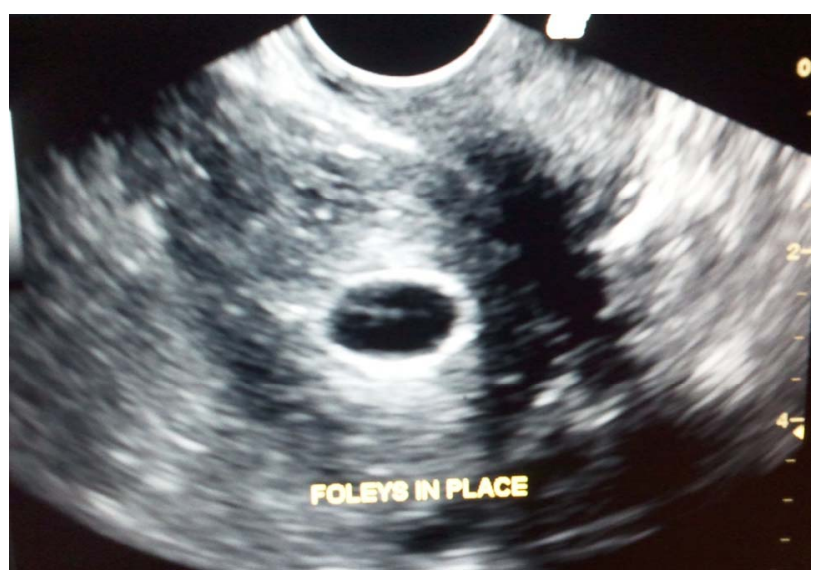

Figure 1. Axial transvaginal USG showing foley's bulb in situ to hold the catheter in place.

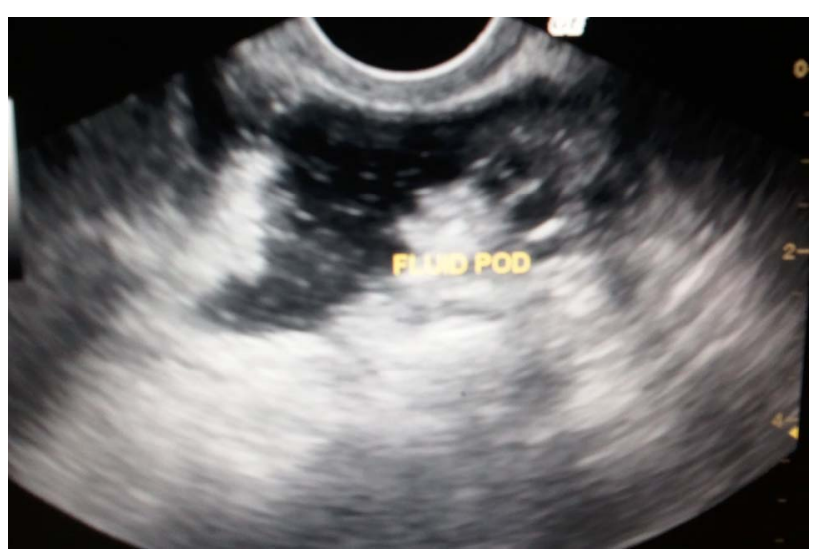

Figure 2. Female patient with secondary infertility. Free spillage of saline was noted real time and fluid seen in the pouch of douglas. followed by chromopertubation. Pathology found during laparoscopy was treated accordingly. Patient was discharged with antibiotic and analgesic cover (Cefixime and Ibuprofen + Paracetamol for 7 days, orally). Stitch removal was done after 7 days.

The patients tolerated the procedure well without any major discomfort. The average pain score in our patients was 2 - 3 with no major complications. Average time to perform the procedure was 10 minutes.

The findings of both modalities were compared. After data analysis statistical parameters were calculated taking laparoscopy as gold standard.

\section{Results}

Majority of patients belongs to 25 to 35 years age group (76\%). Out of 123 patients 49 patients had tubal pathologies on sonosalpingography (Figures 3-5). This included cornual block, unilateral, bilateral, hydrosalpinx, fimbrial adhesions etc. When compared to laparoscopic findings the sensitivity of sonosalpingography was $72.91 \%$, specificity of test was $81.33 \%$. Diagnostic accuracy was found to be $78.05 \%$ and positive predictive value of $71.42 \%$ (Table 1).

\section{Discussion}

In our study we used only gray scale sonosalpingography and focussed the adnexal region. All our patients underwent laparoscopic evaluation on the same day. Laparoscopy has been used as the gold standard for assessment of tubes in the last two decades. However it requires general anaesthesia and carries the risk of surgical complications such as bowel or vascular injury, haemorrhage, infection and post operative discomfort. Through laparoscopy one is equally able to visualise the total pelvic anatomy [1,2]. It is useful in evaluating ovarian disease, genital anomalies, tubal and adnexal competence and evaluate pelvic

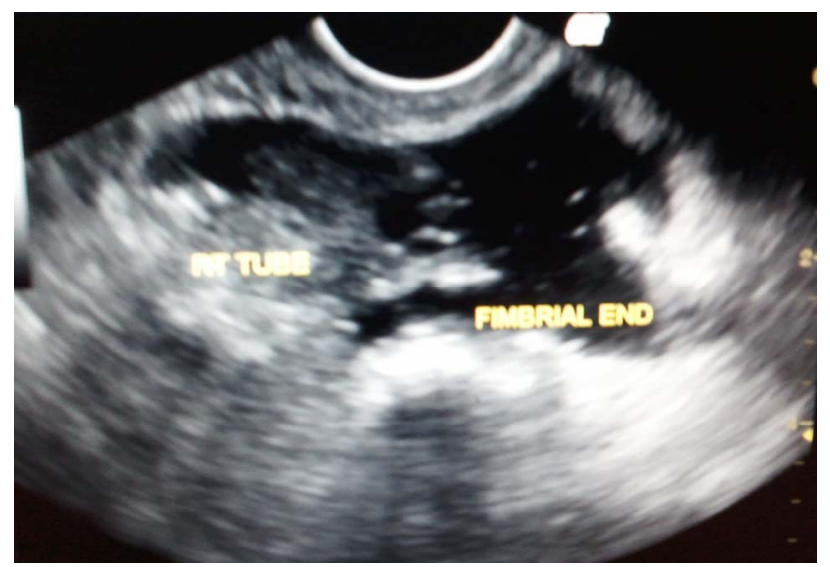

Figure 3. Female with secondary infertility. Axial transvaginal USG shows a patent right fallopian tube visualised till the fimbrial end. 


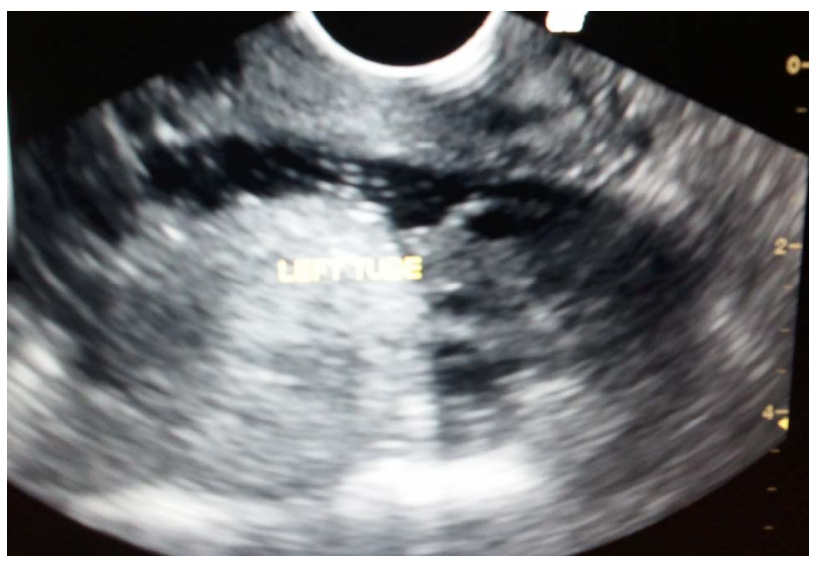

Figure 4. Female with secondary infertility. Axial transvaginal USG shows a patent left fallopian tube visualised till the fimbrial end.

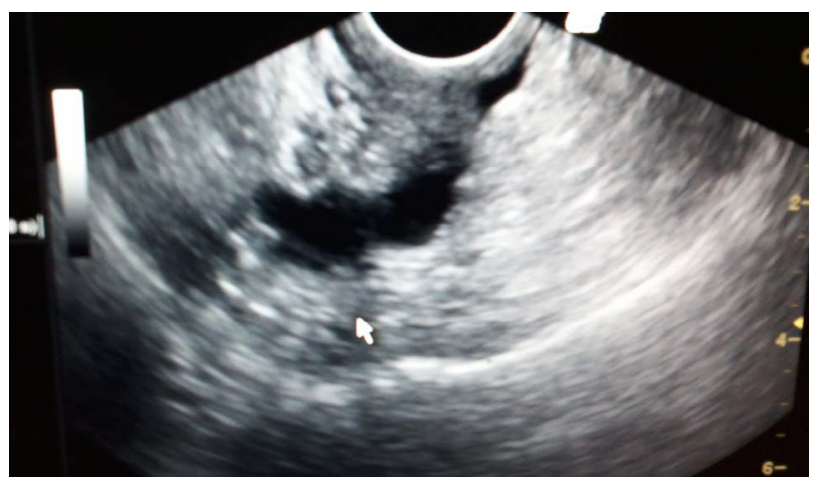

Figure 5. Patient with secondary infertility. Longitudinal transvaginal USG showed hydro-salpinx right side and block at distal aspect probably due to fimbrial adhesions.

Table 1. Sonosalpingography: statistical data for tubal pathology.

\begin{tabular}{cc} 
Total number of patients & 123 \\
True positives & 35 \\
True negatives & 61 \\
False positives & 14 \\
False negatives & 13 \\
Sensitivity & $72.91 \%$ \\
Specificity & $81.33 \%$ \\
Diagnostic accuracy & $78.05 \%$ \\
Positive predictive value & $71.42 \%$ \\
Negative predictive value & $82.43 \%$ \\
\hline
\end{tabular}

distortions. It is valuable to reach an accurate classification of endometriosis of the pelvis. The accuracy of sono HSG compared to X-ray HSG varies from $70.37 \%$ to 92.20\% according to Peters et al. [3] and Volpi et al. [4]. The accuracy of sono HSG compared to chromopertubation is from $81.82 \%$ according to Stern et al. [5] and $91.48 \%$ according to Kupesic et al. [6]. In our study of 123 patients using only gray scale sonosalpingosonography, performed by a single observer the diagnostic accu- racy was $78.05 \%$ for tubal pathologies.

Transvaginal sonosalpingography performed by using isotonic saline without anaesthesia is physiological, easy to perform, safe, cost effective, non invasive and more convenient when compared with other conventional methods, to assess the accuracy of the diagnosis of tubal occlusion [7]. In study of Peters and Coulam [3], results of ultrasonographic HSG were compared with X-ray HSG. 69 of 85 (81\%) patients showed agreement. In 50 of 58 (86\%) patients findings agreed with observations at chromopertubation. The frequency of comparable findings between X-ray HSG and chromopertubation was $75 \%$. Richman and colleagues [8] evaluated tubal patency in 36 infertile women. They compared ultrasound findings with conventional hysterosalpingogram, which had been obtained simultaneously. Ultrasound demonstrated bilateral occlusion with a sensitivity of $100 \%$ and showed tubal patency with a specificity of 96\%. Tufekci and colleagues [9], studied 38 women with infertility. The results obtained from transvaginal hysterosonosalpingography and laparoscopies were completely consistent for 29 cases (76.32\%), and partially consistent for eight cases. Only one case showed inconsistent result. Transvaginal hysterosonosalpingography correctly indicated tubal patency or non patency in 37 of 38 patients. Heikkinen and co-workers [10], evaluated the advantages and accuracy of transvaginal hysterosonosalpingography in the assessment of tubal patency with regards to laparoscopic chromopertubation. Sixty one fallopian tubes were examined by both techniques, resulting in concordance of $85 \%$. On transvaginal sono HSG, 45 tubes were found to be patent and 16 occluded. On chromopertubation, 50 tubes were patent and 11 were occluded. Bilateral tubal patency was found by transvaginal sonosalpingography in 17 cases and by laparoscopy in 22 cases. Bilateral occlusion was found in three cases using either technique.

In our study 35 patients were correctly diagnosed as having tubal block, unilateral/bilateral. Thirteen cases were missed mainly due to tubal spasm and cases of mild to moderate hydrosalpinx. These factors can be seen with any technique. We also missed two cases of tubal dysmotility due to pelvic adhesions secondary to pelvic endometriosis. Transvaginal sono HSG with the combination of air and saline is a low cost, reliable, safe and comfortable examination method and it can be used for the primary investigation of infertility. We completed our examination in an average time of 10 minutes. In one patient procedure was abandoned due to highly stenosed cervix. Contrary to optimistic results of different ultrasound techniques for evaluation of tubal patency, Balen and colleagues [11], found contrast HSG using saline and Echovist contrast media insufficiently accurate and inferior to conventional X-ray HSG. False positive rates in range of $9 \%$ and false negatives rates in range of $20 \%$ have been reported in the 
diagnosis of tubal obstruction by colour Doppler HSG [5].

\section{Conclusion}

Sonosalpingography is a very useful complementary diagnostic modality in assessment of tubal pathology. This should precede direct laparoscopic evaluation. Patients of secondary infertility can be assessed with a combination of sonosalpingography and laparoscopy. This saves the patient of radiation exposure as well as pain of contrast administration.

\section{REFERENCES}

[1] G. M. Arronet, S. Y. Abduljie and I. R. O’Brien, “A Nine Year Survey of Fallopian Tube Dysfunction in Human Infertility: Diagnosis and Therapu,” Fertility and Sterility, Vol. 20, No. 6, 1969, pp. 903-918.

[2] A. S. Thurmond and J. Rosch, "Nonsurgical Fallopian Tube Recanalisation for Treatment of Infertility,” Radiology, Vol. 174, 1990, pp. 371-374.

[3] A. J. Peters and C. B. Coulam, "Hysterosalpingography with Color Doppler Ultrasonography,” American Journal of Obstetrics and Gynecology, Vol. 164, No. 6, 1991, pp. 1530-1534.

[4] E. Volpi, G. Zuccaro, A. Patriarca, S. Rustichelli and P. Sismondi, "Transvaginal Sonography Tubal Patency Testing Air and Saline Solution as Contrast Media in a Routine Infertility Clinic Setting,” Ultrasound in Obstetrics \& Gynecology, Vol. 7, No. 1, 1996, pp. 43-48. doi:10.1046/j.1469-0705.1996.07010043.x

[5] J. Stern, A. J. Peters and C. B. Coulam, "Color Doppler Ultrasonography Assessment of Tubal Patency: A Comparision Study with Traditional Technique,” Fertility and Sterility, Vol. 58, No. 5, 1992, pp. 897-900.

[6] S. Kupesic and A. Kurjak, "Gynaecological Vaginal Sonographic Interventional Procedures-What Does Color Add?” Gunecol Perinatol, Vol. 3, 1994, pp. 57-60.

[7] J. A. Cullinan, J. A. Fleischer and A. L. Arnold, "Sonohysterography: A Technique for Endometrial Evaluation," Radiographics, Vol. 15, 1995, pp. 501-514.

[8] T. S. Richman, G. N. Viscomi, A. de Cherney, M. L. Polan and L. O. Alcebo, "Fallopian Tubal Patency Assessed by Ultrasound Fluid Injection,” Radiology, Vol. 152, 1984, pp. 507-510.

[9] E. C. Tufekci, S. Girit, M. D. Bayirli, F. Durmusoglu and S. Yalti, "Evaluation of Tubal Patency by Transvaginal Sonosalpingography," Fertility and Sterility, Vol. 57, 1992, pp. 336-340.

[10] H. Heikkinen, A. Tekay, E. Volpi, H. Martikainen and P. Jouppila, "Transvaginal Salpingosonography for the Assessment of Tubal Patency in Infertile Women: Methodological and Clinical Experiences,” Fertility and Sterility, Vol. 64, No. 2, 1995, pp. 293-298.

[11] F. G. Balen, C. M. Allen, N. C. Siddle and W. R. Less, "Ultrasound Hysterosalpingography-Evaluation as an Outpatient Procedure,” British Journal of Radiology, Vol. 66, 1993, pp. 592-599. doi:10.1259/0007-1285-66-787-592 